\title{
Genome-wide comparative analysis of NBS- encoding genes in four Gossypium species
}

Liuxin Xiang ${ }^{1,2 \dagger}$, Jinggao $\mathrm{Liu}^{3 \dagger}$, Chaofeng $\mathrm{Wu}^{2}$, Yushan Deng ${ }^{2}$, Chaowei Cai ${ }^{1}$, Xiao Zhang ${ }^{1}$ and Yingfan Cai ${ }^{*}$

\begin{abstract}
Background: Nucleotide binding site (NBS) genes encode a large family of disease resistance (R) proteins in plants. The availability of genomic data of the two diploid cotton species, Gossypium arboreum and Gossypium raimondii, and the two allotetraploid cotton species, Gossypium hirsutum (TM-1) and Gossypium barbadense allow for a more comprehensive and systematic comparative study of NBS-encoding genes to elucidate the mechanisms of cotton disease resistance.

Results: Based on the genome assembly data, 246, 365, 588 and 682 NBS-encoding genes were identified in G. arboreum, G. raimondii, G. hirsutum and G. barbadense, respectively. The distribution of NBS-encoding genes among the chromosomes was nonrandom and uneven, and was tended to form clusters. Gene structure analysis showed that G. arboreum and G. hirsutum possessed a greater proportion of $\mathrm{CN}, \mathrm{CNL}$, and $\mathrm{N}$ genes and a lower proportion of NL, TN and TNL genes compared to that of G. raimondii and G. barbadense, while the percentages of RN and RNL genes remained relatively unchanged. The percentage changes among them were largest for TNL genes, about 7 times. Exon statistics showed that the average exon numbers per NBS gene in G. raimondii and G. barbadense were all greater than that in G. arboretum and G. hirsutum. Phylogenetic analysis revealed that the TIR-NBS genes of G. barbadense were closely related with that of G. raimondii. Sequence similarity analysis showed that diploid cotton G. arboreum possessed a larger proportion of NBS-encoding genes similar to that of allotetraploid cotton G. hirsutum, while diploid G. raimondii possessed a larger proportion of NBS-encoding genes similar to that of allotetraploid cotton G. barbadense. The synteny analysis showed that more NBS genes in G. raimondii and G. arboreum were syntenic with that in G. barbadense and G. hirsutum, respectively.

Conclusions: The structural architectures, amino acid sequence similarities and synteny of NBS-encoding genes between G. arboreum and G. hirsutum, and between G. raimondii and G. barbadense were the highest among comparisons between the diploid and allotetraploid genomes, indicating that G. hirsutum inherited more NBS-encoding genes from G. arboreum, while G. barbadense inherited more NBS-encoding genes from G. raimondii. This asymmetric evolution of NBS-encoding genes may help to explain why G. raimondii and G. barbadense are more resistant to Verticillium wilt, whereas G. arboreum and G. hirsutum are more susceptible to Verticillium wilt. The disease resistances of the allotetraploid cotton were related to their NBS-encoding genes especially in regard from which diploid progenitor they were derived, and the TNL genes may have a significant role in disease resistance to Verticillium wilt in G. raimondii and G. barbadense.
\end{abstract}

Keywords: Gossypium species, NBS-encoding gene, Amino acid sequence similarity, Gene structure, Disease resistance

\footnotetext{
*Correspondence: yingfancai@outlook.com

†Equal contributors

${ }^{1}$ State Key Laboratory of Cotton Biology, College of Life Science, Henan Key Laboratory of Plant Stress Biology, Henan University, Kaifeng, Henan 475004, China

Full list of author information is available at the end of the article
} 


\section{Background}

Cotton is one of the most economically important crop plants in the world and is the most important textile fiber crop worldwide. The most widely cultivated cotton species today are allotetraploid Gossypium hirsutum followed by Gossypium barbadense, both of which are originated from interspecific hybridization between the A-genome species Gossypium arboreum (A2) and the D-genome species Gossypium raimondii (D5) [1].

Verticillium wilt and Fusarium wilt are two of the most destructive diseases in cotton production worldwide. Verticillium wilt is caused by the soilborne fungal pathogen Verticillium dahliae. G. raimondii is nearly immune to the pathogen, and G. barbadense is usually resistant or highly resistant to $V$. dahliae, whereas $G$. arboreum and G. hirsutum are often susceptible to $V$. dahliae [2-8]. Fusarium wilt is caused by another soilborne fungal pathogen Fusarium oxysporum f. sp. Vasinfectum. G. barbadense is often more susceptible to $F$. oxysporum compared to G. arboreum and G. hirsutum [9, 10].

Resistance $(\mathrm{R})$ genes play a central role in recognizing effectors from pathogens and in triggering downstream signaling during plant response to pathogen invasions $[11,12]$. Numerous R genes from many plants have been cloned and characterized over the past few decades [13]. Most of the cloned $\mathrm{R}$ genes are nucleotide-binding sites (NBS) genes containing a NBS domain and constitute one of the largest plant resistance gene family [14, 15]. The NBS domain is part of the larger $\sim 300$ amino acid NB-ARC (Apaf-1, R proteins and CED-4) domain and contains five strictly ordered motifs including P-loop, kinase-2, kinase-3a, GLPL and MHDL $[16,17]$. The NBS region binds and hydrolyzes ATP or GTP, and primarily works as a signal transduction switch following pathogen recognition [17]. NBS-encoding genes usually contain additional domains, TIR (the Toll/interleukin-1 receptor), CC (coiled-coil) or RPW8 (Resistant to powdery mildew in $A$. thaliana) in the $\mathrm{N}$-terminal domain and LRR (leucine-rich repeat) domains in the C-terminal region [18].

NBS-encoding genes can be further classified into two major groups according to the presence or absence of different domains in the $\mathrm{N}$-terminal region. The first group is comprised of proteins carrying TIR and members of the group are named TNL proteins (for TIR-NBS-LRR). The second, non-TIR-NBS-LRR group is usually known as CNL (for CC-NBS-LRR) and RNL (for RPW8-NBS-LRR), because most of its members encode CC or RPW8 in the N-terminal domain [19].

With the availability of genomic data for increasing number of cotton species, NBS-encoding genes could be systematically investigated to elucidate their role in contributing to the differences and relationships of disease resistances among cotton species, and help to decipher the mechanisms of disease resistance in cotton.
The genome sequences of the two diploid cotton species, G. raimondii and G. arboreum, and the two allotetraploid cotton species, G. hirsutum (TM-1) and G. barbadense, have been reported [2, 20-22]. In the present work, the assembled genome sequences were utilized to identify NBS disease resistance genes for the four cotton species. Multiple approaches were utilized to assess these NBS genes' architecture in the genome and their evolutionary history, including the characterization of functional domains, their distributions across the genome, their phylogenetic relationships and so on. The analysis provided genome level insights into disease resistance genes among cotton species, which can help to reveal the mechanism of disease-resistance and accelerate the disease-resistant breeding of cotton.

\section{Results}

Identification and classification of NBS-encoding genes

G. arboreum, G. raimondii, G. hirsutum and G. barbadense were selected to identify and compare NBS-encoding genes in their genomes. Searches with HMMER 3.1b2 in the G. arboreum, G. raimondii, G. hirsutum and G. barbadense genomes resulted in the identification of 246, 365, 588 and 682 NBS genes containing NB-ARC domain, respectively (Table 1 and Additional file 1: Table S1). The two allotetraploid cotton plants possessed almost twice the number of NBS genes compared to the two diploid cotton plants, probably because of hybridization between G. arboreum and $G$. raimondii without gene losses, or hybridization with rapid gene losses followed by gene replication after their divergence from the initial hybrid. It can also be due to the combination of the two processes.

Additional domains, TIR, CC or RPW8 in the N-terminal region and LRR domains in the $\mathrm{C}$-terminal region, were identified in the NBS-encoding genes. If a TIR, CC or RPW8 domain was detected before NB-ARC domain, it was coded as "T", "C", " $R$ ", respectively; if LRR domain was detected after NB-ARC domain, it was coded as "L". The NB-ARC domain was coded as "N". The NBS-encoding genes were sub-classified into eight types according to their domain architecture (Table 1 and Additional file 1: Table S1): CN, CNL, N, NL, RN, RNL, TN and TNL. As shown in Table 1, G. arboreum possessed a larger proportion of $\mathrm{CN}, \mathrm{CNL}$, and $\mathrm{N}$ genes and a lower proportion of NL, TN and TNL genes than G. raimondii. For example, the proportions of $\mathrm{CN}, \mathrm{CNL}$ and N genes of the diploid $\mathrm{G}$. arboreum were 17.89, 32.52 and $23.98 \%$, respectively, while the proportions of $\mathrm{CN}, \mathrm{CNL}$ and $\mathrm{N}$ genes of the diploid G. raimondii were 10.68, 29.32 and 16.99\%, respectively (Table 1). However, the percentage differences of RN or RNL genes between G. arboreum and G. raimondii were less than $1 \%$, so the proportions of RN and RNL genes remained relatively unchanged. Similar results were observed between the two allotetraploid cotton species: 
Table 1 Classification and distribution of NBS-encoding genes in the four cotton genomes

\begin{tabular}{|c|c|c|c|c|c|c|c|c|}
\hline \multirow{2}{*}{$\begin{array}{l}\text { Gene } \\
\text { types }\end{array}$} & \multicolumn{2}{|c|}{ G. arboreum } & \multicolumn{2}{|c|}{ G. raimondii } & \multicolumn{2}{|c|}{ G. hirsutum } & \multicolumn{2}{|c|}{ G. barbadense } \\
\hline & Number & Percentage & Number & Percentage & Number & Percentage & Number & Percentage \\
\hline $\mathrm{CN}$ & 44 & $17.89 \%$ & 39 & $10.68 \%$ & 89 & $15.14 \%$ & 92 & $13.49 \%$ \\
\hline $\mathrm{CNL}$ & 80 & $32.52 \%$ & 107 & $29.32 \%$ & 165 & $28.06 \%$ & 143 & $20.97 \%$ \\
\hline N & 59 & $23.98 \%$ & 62 & $16.99 \%$ & 168 & $28.57 \%$ & 171 & $25.07 \%$ \\
\hline $\mathrm{NL}$ & 53 & $21.54 \%$ & 89 & $24.38 \%$ & 154 & $26.19 \%$ & 210 & $30.79 \%$ \\
\hline RN & 0 & $0.00 \%$ & 1 & $0.27 \%$ & 1 & $0.17 \%$ & 2 & $0.29 \%$ \\
\hline RNL & 3 & $1.22 \%$ & 3 & $0.82 \%$ & 6 & $1.02 \%$ & 9 & $1.32 \%$ \\
\hline TN & 2 & $0.81 \%$ & 14 & $3.84 \%$ & 0 & $0.00 \%$ & 11 & $1.61 \%$ \\
\hline TNL & 5 & $2.03 \%$ & 50 & $13.70 \%$ & 5 & $0.85 \%$ & 44 & $6.45 \%$ \\
\hline Total & 246 & $100.00 \%$ & 365 & $100.00 \%$ & 588 & $100.00 \%$ & 682 & $100.00 \%$ \\
\hline
\end{tabular}

G. hirsutum possessed a larger proportion of $\mathrm{CN}, \mathrm{CNL}$, and $\mathrm{N}$ genes and a lower proportion of NL, TN and TNL genes than G. barbadense, and the proportions of $\mathrm{RN}$ and RNL genes remained relatively unchanged. Therefore, with respect to the proportions of different NBS type genes, G. arboreum and G. hirsutum share similar distribution profiles, while G. raimondii and $G$. barbadense share similar distribution profiles. The results suggest that G. hirsutum may preferentially inherit NBS-encoding genes from G. arboreum progenitor, while G. barbadense may preferentially inherit NBSencoding genes from G. raimondii progenitor. Moreover, the greatest percentage changes between G. raimondii and G. arboreum, and between G. barbadense and G. hirsutum, occurred in the TNL type genes, about 7 times. Therefore, these TNL genes may play a significant role in disease resistance in G. raimondii and G. barbadense.

In addition, the exon number of NBS genes was calculated based on the annotation information of cotton genomes (Additional file 1: Table S1 and Additional file 2: Table S9). NBS genes in G. raimondii and G. barbadense were predicted to have 2.8 and 3.8 exons in average respectively, which was much larger than the average number of NBS genes in G. arboretum and G. hirsutum, 2.3 and 2.2 respectively. The mean exon numbers in eight NBS gene type were quite different in each cotton species, but the exons of NBS genes with TIR or RPW8 domain were generally more than that of other NBS type genes, especially TNL type genes containing mean 4.4 to 9.5 exons. The larger exon number in cotton TNL type genes was consistent with the results of a previous study in the Arabidopsis, poplar, grapevine and Rosaceae $[17,23,24]$.

\section{Chromosome location and gene cluster identification}

Based on the location of individual NBS genes, 246 NBS genes and 273 of the 365 NBS genes, were mapped on the 13 chromosomes of G. arboreum (Chr A01-13, Fig. 1a) and G. raimondii (Chr D01-13, Fig. 1b), respectively; 311 of the 588 NBS genes and 395 of the 682 NBS genes were mapped on the 26 chromosomes (Chr A01-13 and Chr D01-13) of G. hirsutum and G. barbadense, respectively (Additional file 3: Figure S1A and B). The remaining NBS genes were located on other scaffolds that had not been yet linked to a chromosome (Additional file 1: Table S1).

The distribution of NBS-encoding genes was nonrandom among the chromosomes (Fig. 1, Additional file 3: Figure S1, Additional file 1: Table S1, and Additional file 4: Table S2). For example, G.arboreum chromosome A04 contained the greatest number (56) of NBS-encoding genes, including 6 of the 8 NBS gene types $(\mathrm{N}, \mathrm{NL}, \mathrm{CN}$, CNL, TN and TNL); whereas only four NBS-encoding genes were located on chromosome A03 which contained 3 NBS gene types (NL, CN and CNL). G. raimondii chromosome D07 contained the greatest number (85) of NBS-encoding genes, including 7 of the 8 NBS gene types $(\mathrm{N}, \mathrm{NL}, \mathrm{CN}, \mathrm{CNL}, \mathrm{TN}, \mathrm{TNL}$ and $\mathrm{RN}$ ); whereas only one NBS-encoding gene was located on chromosome D12. G. hirsutum chromosome D09 contained the greatest number (86) of NBS-encoding genes, including 5 NBS gene types $(\mathrm{N}, \mathrm{NL}, \mathrm{CN}, \mathrm{CNL}$ and $\mathrm{RNL})$; whereas no NBS-encoding gene was located on chromosome D12. G. barbadense chromosome A11 contained the greatest number (70) of NBS-encoding genes, including 7 NBS gene types $(\mathrm{N}, \mathrm{NL}, \mathrm{CN}, \mathrm{CNL}, \mathrm{TN}, \mathrm{TNL}$ and $\mathrm{RNL})$; whereas no NBS-encoding gene was located on chromosome D04.

Moreover, the distribution of NBS-encoding genes was not even within the chromosomes and they tended to form clusters in other plants (tandem repeats [25-30]) (Table 2). This clustered arrangement has been thought to facilitate sequence exchange through recombinational mispairing [31]. Studies of NBS-encoding genes in Arabidopsis, rice, sorghum and maize also indicated uneven distributions on chromosomes and showed that most NBS-encoding genes were found in clusters [25-27, 32]. To identify NBS gene clusters, we used a 


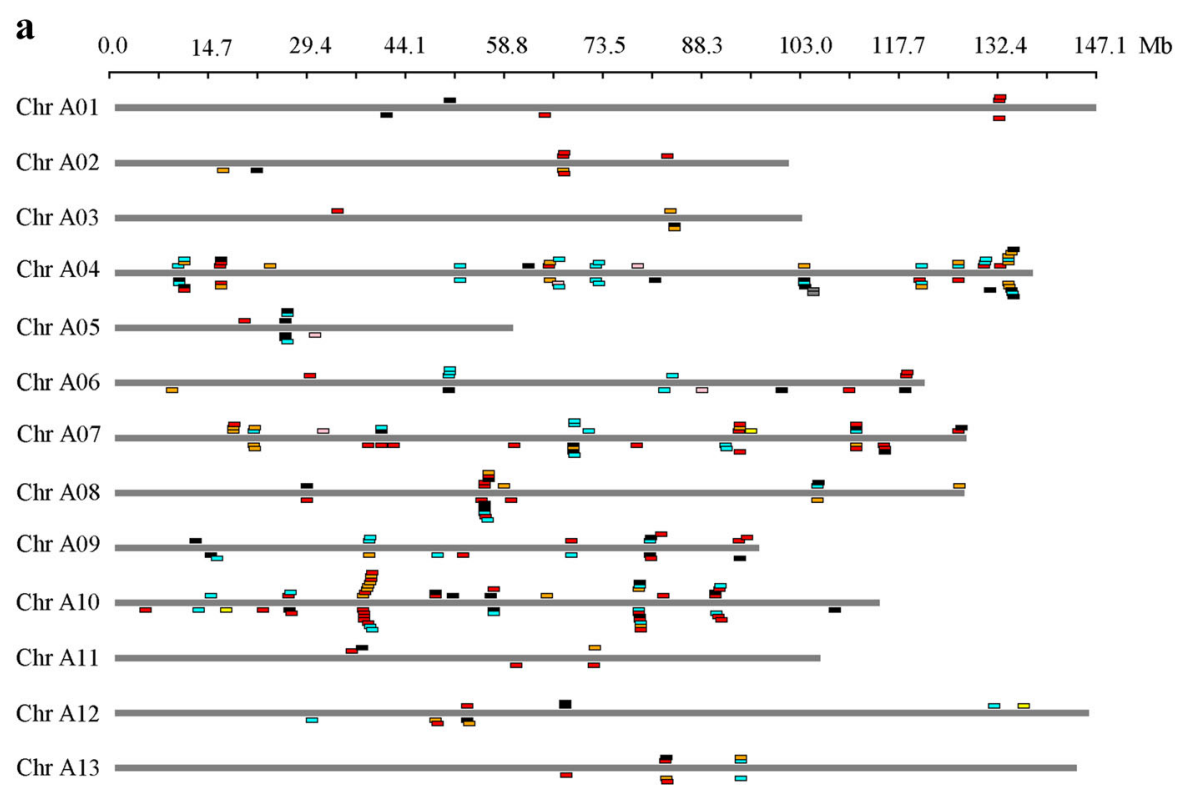

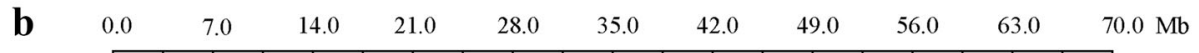

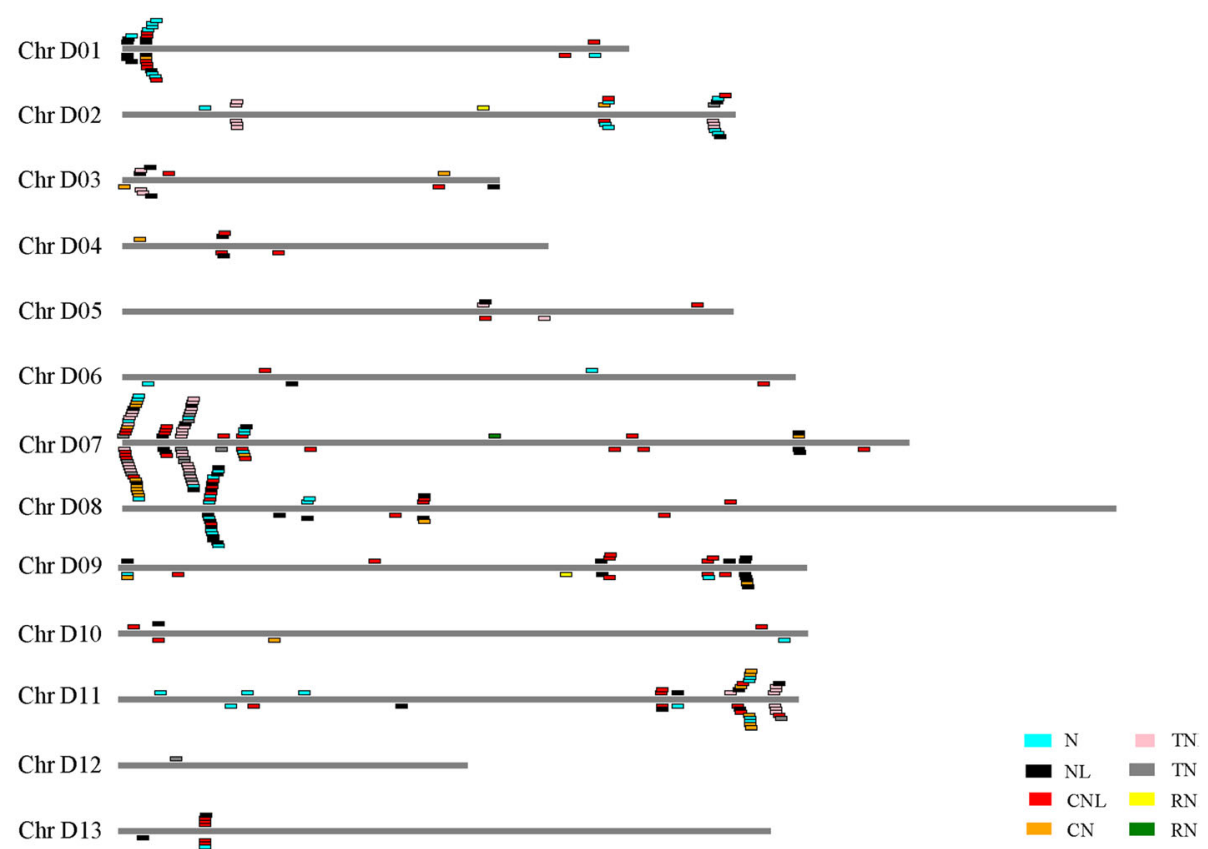

Fig. 1 Distribution of NBS-encoding genes in chromosomes. a Distribution in G. arboreum chromosomes. b Distribution in G. raimondii chromosomes

previous definition [33] that a gene cluster was considered a chromosome or a scaffold region when such region contained two or more genes within $200 \mathrm{~kb}$. Using this criteria, we identified 54, 52, 88 and 147 clusters containing 182, 287, 463 and 520 NBS genes in G. arboreum, G. raimondii, G. hirsutum and G. barbadense, respectively (Table 2, Additional file 1: Table S1, and Additional file 4: Table S2). The size of the clusters varied across the genome from 2 to 86 members (Additional file 4: Table S2). Each cluster had an average of about 3.4, 5.5, 5.3 and 3.5 genes and about 74.0, 78.6, 78.7 and 76.2\% of NBS genes occurred in clusters in G. arboreum, G. raimondii, G. hirsutum and G. barbadense, respectively (Table 2). These results indicate that NBS-encoding genes of the four cotton species also tend to form clusters and the average NBS gene numbers per cluster among species were different (Table 2). 
Table 2 Organization of NBS-encoding genes in ten plant genomes

\begin{tabular}{|c|c|c|c|c|c|}
\hline Plant species & No. of NBS genes & No. of cluster genes & Proportion of cluster genes & No. of clusters & Mean members per cluster \\
\hline Gossypium arboreum & 246 & 182 & $74.0 \%$ & 54 & 3.4 \\
\hline Gossypium raimondii & 365 & 287 & $78.6 \%$ & 52 & 5.5 \\
\hline Gossypium hirsutum & 588 & 463 & $78.7 \%$ & 88 & 5.3 \\
\hline Gossypium barbadense & 682 & 520 & $76.2 \%$ & 147 & 3.5 \\
\hline Theobroma cacao & 298 & 241 & $80.1 \%$ & 52 & 4.6 \\
\hline Zea mays $\left(\right.$ Maize) ${ }^{a}$ & 107 & 61 & $57.0 \%$ & 22 & 2.8 \\
\hline Sorghum bicolor ${ }^{a}$ & 236 & 217 & $91.9 \%$ & 25 & 8.7 \\
\hline Oryza sativa (Rice) ${ }^{a}$ & 519 & 362 & $69.7 \%$ & 104 & 3.5 \\
\hline Arabidopsis thaliana $^{\mathrm{a}}$ & 171 & 125 & $73.1 \%$ & 39 & 3.2 \\
\hline Medicago truncatula ${ }^{a}$ & 469 & 310 & $66.1 \%$ & 61 & 5.1 \\
\hline
\end{tabular}

${ }^{a}$ Data from Cheng Y et al. [25]

Interestingly, though the total numbers of NBS-encoding genes among the four cotton species were quite different, their proportions of cluster genes remained relatively unchanged, 74-79\% (Table 2). These proportions are greatly different from those of maize (57.0\%) and sorghum (91.9\%), but only slightly different from those of rice, Arabidopsis or Medicago truncatula (Table 2) [25]. We also identified 298 NBS-encoding genes and 52 NBS clusters in the genome of Theobroma cacao, a close relative of cotton (Additional file 1: Table S1). The 52 NBS clusters contained 241 NBS genes averaging 4.6 genes per cluster. About $80.1 \%$ of NBS genes occurred in clusters. Apparently, the proportion of NBS cluster genes in cacao was closer to that in cotton than to that in the other crops such as maize and sorghum. It was speculated that the duplication and clustering of NBS genes may be related to species evolution.

\section{Similarity analysis of NBS-encoding genes among the four cotton species}

Allotetraploid G. hirsutum and G. barbadense originated from interspecific hybridization between the A-genome species G. arboreum (A2) and the D-genome species G. raimondii (D5) [1]. To further elucidate the evolutionary relationship of NBS-encoding genes between the allotetraploid and the diploid cotton species, the NBSencoding proteins with sequence similarity greater than $90,80,70,60,50,40$ and $30 \%$ between the allotetraploid and diploid cotton species were identified (Additional file 5: Table S3, Additional file 6: Table S4, Table Additional file 7: Table S5, Additional file 8: Table S6), respectively. The numbers of non-redundant NBS-encoding genes under different similarity level in G. hirsutum, G. barbadense, G. arboreum and G. raimondii were then calculated, respectively (Table 3 ). The results revealed that the number of non-redundant NBS-encoding genes in G. hirsutum or G. barbadense increased with decreasing sequence similarity, but the amount of increases became smaller (Table 3). For sequence similarity greater than $70 \%$, the proportion of NBS-encoding genes in G. hirsutum or G. barbadense was greater than $75 \%$. For sequence similarity greater than 50\%, the proportion of NBS-encoding genes in G. hirsutum or G. barbadense was greater than $90 \%$. Therefore, NBS-encoding genes of the allotetraploid cotton had high amino acid sequence similarity with that of the diploid cotton.

The numbers of NBS-encoding genes in G. hirsutum and $G$. barbadense were 588 and 682, respectively; about two times of the number of NBS-encoding genes in $G$. arboreum and G. raimondii, 246 and 365, respectively. However, the disease resistances of G. hirsutum and G. barbadense were not always stronger than G. arboreum and $G$. raimondii. The reason may be that most of NBSencoding genes of $G$. hirsutum and $G$. barbadense had high amino acid sequence similarity with that of $G$. arboreum and G. raimondii, respectively (Table 3). Comparing sequence similarities between G. hirsutum and the two diploid cotton species, as signed with the solid triangles in Table 3, revealed that the NBS-encoding gene proportion of G. arboreum was greater than that of G. raimondii in G. hirsutum at all the sequence similarity levels. For example, $82 \%$ of NBS-encoding genes of G. arboreum compared to only $71 \%$ of the genes of G. raimondii had orthologous in G. hirsutum at greater than $80 \%$ sequence similarity level, indicating that G. hirsutum may inherit more NBS-encoding genes from G. arboreum than from G. raimondii. Similarly, comparing the sequence similarities between $G$. barbadense and the two diploid cotton species, as signed with the solid squares in Table 3, revealed that the NBS-encoding gene proportion of $G$. raimondii was greater than that of G. arboreum at all the sequence similarity levels. For example, $85 \%$ of NBS-encoding genes of G. raimondii compared to only $75 \%$ of the genes of G. arboreum had 


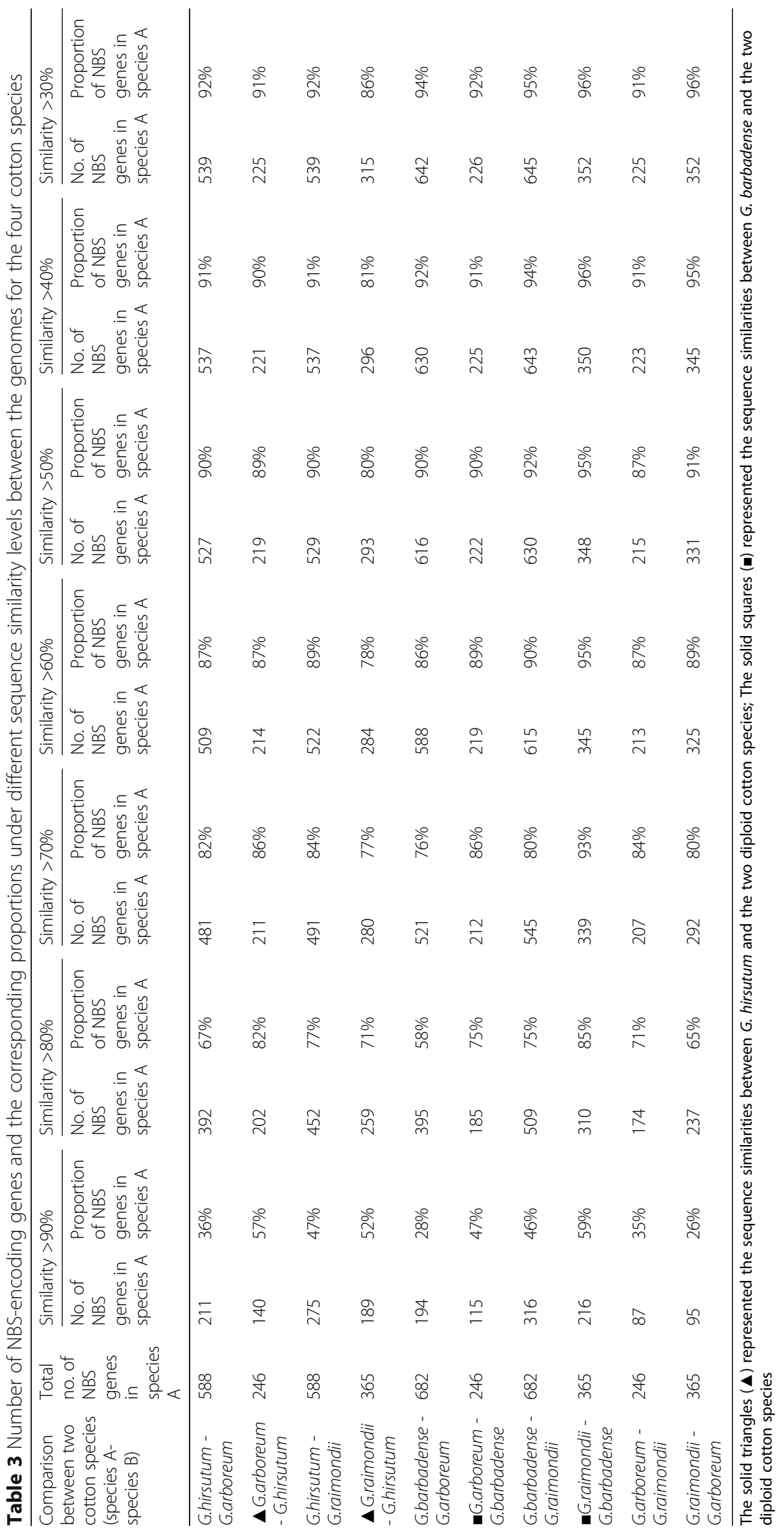


orthologous in G. barbadense at greater than $80 \%$ sequence similarity level, indicating that G. barbadense may inherit more NBS-encoding genes from G. raimondii than from $G$. arboreum. These evidences further support the findings of closely genetic relationships between G. hirsutum and G. arboreum, and between G. barbadense and G. raimondii from the study of the proportion comparisons of different NBS type genes in cotton species (Table 1).

The NBS-encoding proteins with sequence similarity greater than $90,80,70,60,50,40$ and $30 \%$ between G. arboreum and G. raimondii were also identified (Additional file 9: Table S7), respectively and the corresponding numbers of non-redundant NBS genes were calculated (Table 3). The proportion of similar NBS-encoding genes between G. arboreum and G. raimondii was also high (Table 3 ). For sequence similarity greater than $70 \%$, the NBS-encoding gene proportions of G. arboreum and G. raimondii were more than $80 \%$, indicating that these NBS-encoding genes were homogenous, and more tandem duplications of NBS-encoding genes may occur in G. raimondii than in G. arboreum after their divergence. There were 21 and 13 NBS genes (Additional file 10: Table S8) whose sequence similarity were less than $30 \%$ between the two genomes of G. arboreum and G. raimondii, respectively and they were all $\mathrm{N}$ type genes. These 21 and 13 NBS genes may play important roles in providing different disease resistance capabilities in G. arboreum and G. raimondii, respectively.

\section{Synteny analysis of NBS genes between diploid and allotetraploid cotton}

The synteny of genes across several plant species could provide insights to its evolution. The synteny analysis of NBS genes between diploid and allotetraploid cotton was conducted using McScanX (Fig. 2). There were 50, 30, 27, 20 syntenic blocks of NBS genes containing 378, 227, 189 and 154 collinear gene pairs between G. raimondii and G. barbadense, between G. arboreum and G. hirsutum, between G. raimondii and G. hirsutum, and between G. arboreum and G. barbadense, respectively (Additional file 11: Table S10). 157 (43\%) and 88 (24.1\%) NBS genes in G. raimondii were syntenic with that in G. barbadense and G. hirsutum respectively, while 115 (46.7\%) and 62 (25.2\%) NBS genes in G. arboretum were syntenic with that in G. hirsutum and G. barbadense, respectively. The results showed that more NBS genes in G. raimondii and G. arboreum were syntenic with that in G. barbadense and G. hirsutum respectively, indicating that the NBS genes between G. raimondii and G. barbadense, and between G. arboreum and G. hirsutum possessed closer evolution relationships, which was consistent with the results of similarity analysis and structural architectures analysis.

\section{Phylogenetic analysis of NBS-encoding genes containing TIR domain}

The amino acid sequences of the 131 NBS-encoding genes containing TIR domain (TIR-NBS gene) from $G$. arboreum, G. raimondii, G. hirsutum and G. barbadense were aligned, and a phylogenetic tree was generated by the Neighbor-joining method (Fig. 3).

Phylogenetic reconstruction of these TIR-NBS genes showed that the genes from the same cotton species were not always clustered together, but often scattered in different clades (Fig. 3). For example, the 7 TIR-NBS genes from G. arboreum were distributed in separate clades and were distantly related with each other. This result indicated that TIR-NBS genes among four cotton species were homologous. As shown in Fig. 3, Ga044_A04 gene in G. arboreum, and Gr115_D07, Gr116_D07, Gr117_D07 and Gr118_D07 genes in G. raimondii shared a common ancestor. Gr115_D07, Gr116_D07, Gr117_D07 and Gr118_D07 genes belonged to a gene cluster (Additional file 1: Table S1), indicating that tandem duplications of TIR-NBS genes occurred in G. raimondii after its divergence from $G$. arboreum. Except Gb434_scaffold12708 and Gb146_A10 which formed lone clades, the TIR-NBS genes of G. barbadense were closely related with that of G. raimondii (Fig. 3). For example, there were many sister genes between $G$. raimondii and G. barbadense, Gb399_scaffold10391 and Gr123_D07 genes, Gb469_scaffold15653 and Gr119_D07 genes, Gb142_A10 and Gr028_D02 genes and so on. This result indicated that G. barbadense inherited many TIR-NBS genes from G. raimondii.

\section{Discussion}

The NBS-encoding gene family is the largest disease resistance gene family in plants and has been studied in many important plant species, including Arabidopsis thaliana [34], Oryza sativa [32], Medicago truncatula [35], Zea mays [25], and Sorghum bicolor [26]. Cotton is the most important textile fiber crop worldwide. Verticillium wilt and Fusarium wilt are the main threats to cotton production, and the immunity or resistance levels to the diseases differed among cotton species. Little is known about the mechanisms of resistance to Verticillium wilt and Fusarium wilt. To date, no comprehensive and systematic research has been conducted on the NBS-encoding genes in the four important species of the genus Gossypium. The present comparative study on genome-wide analysis of NBSencoding genes in the four Gossypium species provided new insights and useful information.

Our bioinformatics analyses identified 246, 365, 588 and 682 NBS-encoding genes in G. arboreum, G. raimondii, G. hirsutum and G. barbadense, respectively. A different number, 355 NBS-encoding genes identified from G. raimondii by Wei et al. [36] may be due to the 


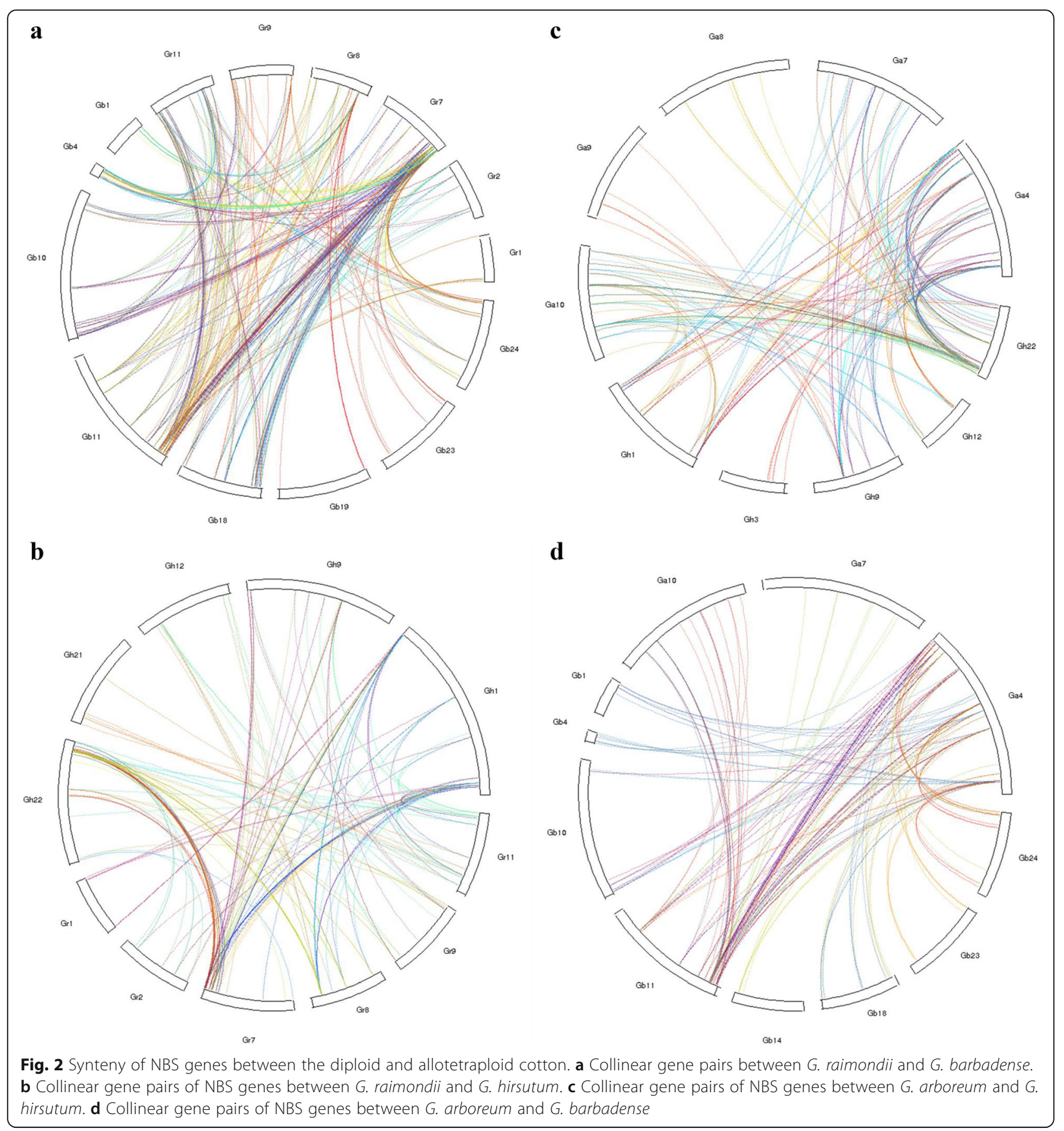

different criterions and a few genes containing NBS domain may be filtered out by programming firstly the homology alignment of protein sequences between all genes in G. raimondii and 113 reference disease resistance genes selected from plant resistance gene database [36]. The numbers of NBS-encoding genes of G. hirsutum and G. barbadense were about two times of that of G. arboreum and G. raimondii, but their disease resistance were not always stronger than that of G. arboreum and G. raimondii. It is suggested that the total number of NBS-encoding genes in the genome is not the decisive factor for the disease resistance in cotton. For example, G. raimondii is more resistant to Verticillium wilt than G. hirsutum [3-8], while G. arboreum is more resistant to Fusarium wilt than G. barbadense [9, 10]. Through the analysis of some resistance gene analogues encoding NBS-LRR domains in cotton, Khan et al. demonstrated that evolution and variation of NBS-LRR genes is one of the reasons for the susceptibility of G. hirsutum to the pathogens as compared to its donor parents [37]. The 


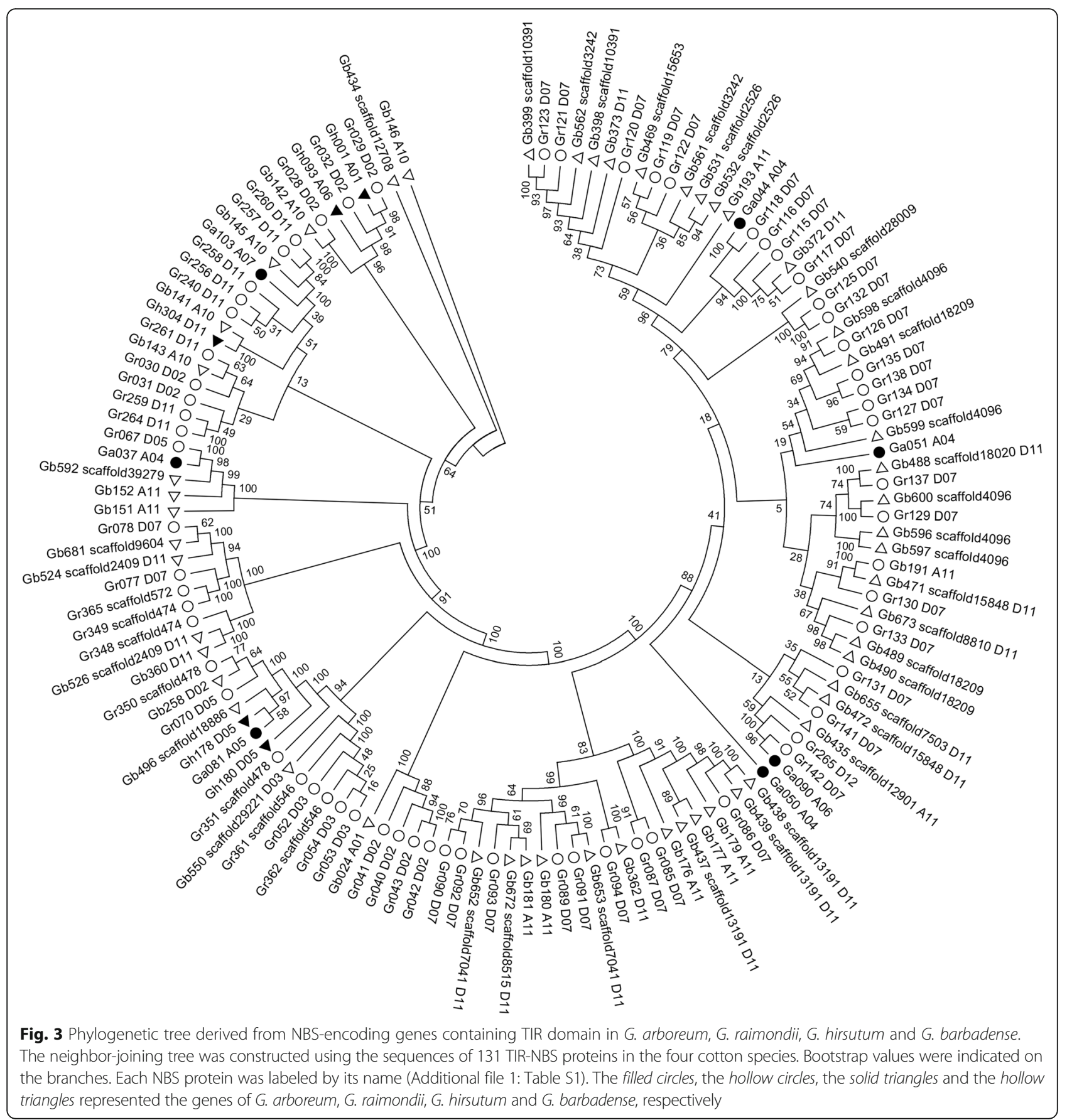

evolution and variation of NBS-encoding genes were selected for the survival of the allotetraploid cotton, so their resistances to different diseases were distinct. For example, G. barbadense usually is resistant or highly resistant to Verticillium wilt, whereas G. hirsutum is susceptible [3-8]; G. barbadense is more susceptible to Fusarium wilt than G. hirsutum $[9,10]$. In our analysis, the disease resistances of the allotetraploid cotton were more closely related to the preferential adoption and duplications of their NBS-encoding genes from one of the two diploid parents. Thus, the comparative analysis of the proportions of different NBS type genes showed that G. arboreum and G. hirsutum had more NBS-encoding genes in common, while G. raimondii and G. barbadense had more NBS-encoding genes in common. For CN, $\mathrm{CNL}$ and $\mathrm{N}$ genes, the diploid G. arboreum had a greater proportion than the diploid G. raimondii, and the allotetraploid G. hirsutum had a greater proportion than the allotetraploid G. barbadense. Opposite results were observed for the NL, TN and TNL genes. The proportions 
of RN and RNL genes remained relatively unchanged. Exon statistics also showed that NBS genes in G. raimondii and G. barbadense generally possessed more exons than that in G. arboretum and G. hirsutum. Therefore, G. hirsutum may inherit more NBS-encoding genes from G. arboreum, while G. barbadense may inherit more NBS-encoding genes from G. raimondii. We found large proportion percentage changes of TNL and TN genes within the two diploid species and within the two allotetraploid species, especially TNL genes, indicating that TNL genes may have a significant role in disease resistance in G. raimondii and G. hirsutum. Li F et al. found similar differences in TNL and TN genes between G. arboreum and G. raimondii, and quantitative RT-PCR (qRT-PCR) analysis of the TNL and TN genes upon $V$. dahliae infection showed that there were different degrees of expression in the G. raimondii [2].

Our phylogenetic analysis of TNL and TN genes (TIR-NBS genes) revealed that the TIR-NBS genes of G. barbadense were closely related to that of G. raimondii, and G. barbadense inherited many TIR-NBS genes from G. raimondii. Further similarity analysis of NBS-encoding genes in the four cotton species showed that G. arboreum had a larger proportion of NBS-encoding genes similar to that of G. hirsutum, and G. raimondii had a larger proportion of NBS-encoding genes similar to that of $G$. barbadense, indicating that G. hirsutum may inherit more NBS-encoding genes from G. arboreum, while G. barbadense may inherit more NBS-encoding genes from $G$. raimondii. The synteny analysis showed that more NBS genes in G. raimondii and G. arboreum were syntenic with that in G. barbadense and G. hirsutum respectively, indicating that the NBS genes between G. raimondii and G. barbadense, and between G. arboreum and G. hirsutum possessed closer evolution relationships. These results are consistent with the research reported by He L et al. [38], Zhang T et al. [39], and Liu X et al. [40]. He L et al. demonstrated that the distribution of resistance gene analogue (RGA) of G. hirsutum between the two sub-genomes A and D of cotton was uneven, with RGA being more abundant in the A sub-genome than in the D sub-genome [38]. Zhang $\mathrm{T}$ et al. found that structural rearrangements, gene loss, disrupted genes and sequence divergence of G. hirsutum were more common in the A sub-genome than in the D sub-genome [39]. Liu X et al. revealed that $\mathrm{A}$ and $\mathrm{D}$ sub-genomes of G. barbadense had a high level of co-linearity with the G. raimondii genome [40]. The present comparative study of disease resistance in cotton also showed that G. raimondii and G. barbadense are immune or resistant to Verticillium wilt, whereas G. arboreum and G. hirsutum are often susceptible to the wilt [2-8]; G. barbadense is often more susceptible to Fusarium wilt than G. arboreum and G. hirsutum $[9,10]$. Therefore, we propose that $G$. hirsutum inherited more NBS-encoding genes from G. arboreum, while G. barbadense inherited more NBS-encoding genes from G. raimondii, suggesting an asymmetric evolution of NBS-encoding genes. This will help to explain why $G$. raimondii and $G$. barbadense are similarly immune to Verticillium wilt, whereas G. arboreum and G. hirsutum are both susceptible to the wilt. Our study will help to reveal the mechanism of disease-resistance and promote the diseaseresistant breeding to improve cotton disease resistance.

\section{Conclusions}

In all, 246, 365, 588 and 682 NBS-encoding genes were identified in G. arboreum, G. raimondii, G. hirsutum and G. barbadense, respectively. The NBS-encoding genes tended to form clusters on chromosomes. There were many commonality of structural architecture, synteny and amino acid sequence similarity of NBS-encoding genes between G. arboreum and G. hirsutum, and between G. raimondii and G. barbadense, indicating that G. hirsutum inherited more NBS-encoding genes from G. arboreum, while G. barbadense inherited more NBS-encoding genes from G. raimondii. This suggests asymmetric evolution of NBS-encoding genes in the two allotetraploid cotton species. The number of NBS-encoding genes is not the decisive factor of disease resistance in cotton, and the disease resistances of the allotetraploid cotton is related to the preferential adoption and duplication of their NBS-encoding genes from one of the two diploid parents. This will help to explain why G. raimondii and G. barbadense are similarly immune or resistant to Verticillium wilt, whereas G. arboreum and G. hirsutum are similarly susceptible to the wilt, and the TNL genes may have a significant role in Verticillium wilt resistance in G. raimondii and G. barbadense.

\section{Methods \\ Cotton genome resources}

Four whole-genome sequenced cotton plants were used in the present study, including two diploid cottons (Gossypium raimondii and Gossypium arboreum) and two allotetraploid cottons (Gossypium hirsutum and Gossypium barbadense). G. raimondii, G. arboreum and G. hirsutum gene information was provided by the Cotton Research Institute, Chinese Academy of Agricultural Sciences (http://cgp.genomics.org.cn/), while G. barbadense gene information was provided by College of Plant Science and Technology \& Group of Cotton Genetic Improvement, Huazhong Agricultural University (http:// cotton.cropdb.org/cotton/). The information contains annotations of 40,976, 4,0134, 76,943, 109,918 protein-coding genes in the G.raimondii, G. arboreum, G. hirsutum and G. barbadense genomes, respectively. The genome sequences of Theobroma cacao were downloaded from 
CocoaGen DB (http://cocoagendb.cirad.fr./) which contained 46,143 protein sequences.

\section{Identification and classification of NBS-encoding genes} All predicted protein sequences from the cotton genomes and cacao genome were scanned with HMMER $3.1 \mathrm{~b} 2$ [41] using the Hidden Markov Model (HMM) corresponding to the Pfam database (profile HMM library) (http://pfam.xfam.org/search\#tabview=tab1; Expect value cut-off of default gathering threshold: the minimum score a sequence must attain in order to belong to the full alignment of a Pfam entry). All genes that contained NB-ARC domains (Pfam: PF00931) by the Pfam search were selected and considered as the NBS-encoding genes.

NBS-encoding genes usually have additional domains such as TIR, CC or RPW8 in the N-terminal domain and a variable number of LRR domains in the carboxyterminal region [18]. The additional domains of these cotton NBS-encoding genes were also identified. The CC domain was detected using HMMER 3.1b2 [41] (https://www.ebi.ac.uk/Tools/hmmer/search/hmmscan) and the results were confirmed using program COILS (http://www.ch.embnet.org/software/COILS_form.html) with default parameters and 0.9 threshold [34]. The TIR, RPW8 and LRR domains were identified using the NCBI Conserved Domains Tool (https://www.ncbi.nlm.nih. gov/Structure/bwrpsb/bwrpsb.cgi; Expect value cut-off of default threshold: 0.01). All the sequences were searched against following databases contained in the NCBI Conserved Domains Tool: CDD v3.15, Pfam v28.0, SMART v6.0, KOG v1.0, COG v1.0, PRK v6.9 and TIGR v15.0. The following accession numbers were found for TIR: pfam01582, cl23801 and smart00255; for RPW8: pfam05659, and cl05301; and for LRR: pfam00560, pfam07723, pfam07725, pfam12799, pfam13306, pfam13516, pfam13855, pfam14850, cl19480, smart00370 and COG4886.

The annotation information of generic feature format (GFF) files for the genomes of four cotton species were used to calculate the exon number of NBS genes.

\section{Genome mapping and gene cluster analysis of NBS- encoding genes}

Using positional information on chromosomes, cotton NBS-encoding genes' physical positions were drawn with GenomePixelizer_October_01_2003 (http://niblrrs. ucdavis.edu/GenomePixelizer/GenomePixelizer_Welcome. html).

Previous studies showed that most NBS-encoding genes were arranged in clusters [25-27, 32]. Therefore, the clusters of NBS-encoding genes in cotton and cacao were identified based on the previously established criteria which considered a chromosome or a scaffold region as a gene cluster when two or more genes were located within a $200 \mathrm{~kb}$ region [33]. For example, for two adjacent genes in a chromosome or a scaffold, if the region between the first gene's start location and the second gene's the end location was less than $200 \mathrm{~kb}$, then the two adjacent genes constitute a cluster.

\section{Alignment and similarity analysis of NBS-encoding proteins}

Multiple alignments of G. raimondii, G. arboreum, G. hirsutum and G. barbadense NBS-encoding protein sequences were performed together using ClustalW [42] with default options and a nj format file containing genetic distance and matched length of any two sequences among all NBS-encoding proteins was generated. One minus the genetic distance was the similarity of two sequences. Considering amino acid sequence length of NB-ARC domain is about 300, two NBS-encoding gene sequences whose matched length was more than 200 amino acids were analyzed subsequently. First, the NBSencoding genes whose protein sequence similarity is more than $90 \%$, more than $80 \%$, more than $70 \%$, more than $60 \%$, more than $50 \%$, more than $40 \%$, and more than $30 \%$ between G. arboreum and G. raimondii were identified using perl program, respectively. Then, the numbers of non-redundant NBS-encoding genes of $G$. arboreum and G. raimondii under different sequence similarity level were calculated, respectively. Similarly, the NBS-encoding genes under different sequence similarity level between G. hirsutum and G. arboreum, between G. hirsutum and G. raimondii, between G. barbadense and G. arboreum, and between G. barbadense and G. raimondii were also identified, respectively. The corresponding numbers of non-redundant NBS-encoding genes of cotton species under different sequence similarity level were then calculated.

\section{Synteny analysis of NBS genes between diploid and allotetraploid cotton}

An BLASTP comparison with e-value 1e-05 using blast2.2.24+ downloaded from NCBI provided the pairwise gene information ( $\mathrm{m} 8$ format) between a diploid and a allotetraploid. According to the BLASTP output, the synteny analysis was constructed using McScanX (http:// chibba.pgml.uga.edu/mcscan2/; default parameters) [43].

\section{Phylogenetic analysis of NBS-encoding genes containing TIR domain}

Multiple alignments of G. raimondii, G. arboreum, G. hirsutum and G. barbadense NBS-encoding protein sequences containing TIR domain (TIR-NBS gene) were performed together using ClustalW [42]. A subsequent manual alignment correction was accomplished by using MEGA 5.05 [44]. Phylogenetic trees were constructed by means of the bootstrap neighbor-joining (NJ) method and the Kimura 2-parameter model that were provided 
by MEGA 5.05 . The stability of internal nodes was assessed by bootstrap analysis with 1000 replicates. These trees were subsequently used to analyze the evolutionary relationships among TIR-NBS genes.

\section{Additional files}

Additional file 1: Table S1. NBS-encoding genes information of $G$. arboreum, G. raimondii, G. hirsutum, G. barbadense and T. cacao. (XLS $495 \mathrm{~kb}$ ) Additional file 2: Table S9. Exon statistics in NBS genes and each NBS gene type in the four cotton species. (XLSX $9 \mathrm{~kb}$ )

Additional file 3: Figure S1. Chromosomal distribution of NBS genes in G. hirsutum and G. barbadense. A. Chromosomal distribution of NBS genes in G. hirsutum. B. Chromosomal distribution of NBS genes in $G$. barbadense. (DOC $3922 \mathrm{~kb}$ )

Additional file 4: Table S2. Information of NBS-encoding gene clusters of G. arboreum, G. raimondii, G. hirsutum and G. barbadense. (XLS $22 \mathrm{~kb}$ )

Additional file 5: Table S3. The amino acid sequence similarity between G. arboretum NBS genes and G. hirsutum NBS genes. (XLS 1623 kb)

Additional file 6: Table S4. The amino acid sequence similarity between G. raimondii NBS genes and G. hirsutum NBS genes. (XLS $2569 \mathrm{~kb}$ )

Additional file 7: Table S5. The amino acid sequence similarity between G. arboretum NBS genes and G. barbadense NBS genes. (XLS 1475 kb)

Additional file 8: Table S6. The amino acid sequence similarity between G. raimondii NBS genes and G. barbadense NBS genes. (XLS 3027 kb)

Additional file 9: Table S7. The amino acid sequence similarity between G. arboretum NBS genes and G. raimondii NBS genes. (XLS $409 \mathrm{~kb}$ )

Additional file 10: Table S8. The NBS-encoding genes whose amino acid sequence similarity less than $30 \%$ between G. arboretum and G. raimondii. (XLS $14 \mathrm{~kb})$

Additional file 11: Table S10. Synteny statistics of NBS genes between the diploid and allotetraploid cotton. (XLS $73 \mathrm{~kb}$ )

\section{Abbreviations}

CC: Coiled-coil; Chr: Chromosome; CN: CC-NBS; CNL: CC-NBS-LRR; LRR: Leucine-rich repeat; NBS: Nucleotide binding site; NL: NBS-LRR; R: Resistance; RN: RPW8-NBS; RNL: RPW8-NBS-LRR; RPW8: Resistant to powdery mildew in A. thaliana; TIR: Toll/interleukin-1 receptor; TN: TIR-NBS; TNL: TIR-NBS-LRR

\section{Acknowledgements}

The authors thank Dr. Lin Bai for checking and suggestions.

\section{Funding}

This work was financially supported by the National Key Research and Development Program of China (2016YFD0101900), the National Science Foundation of China $(31571724,31071461,31470354)$, the National Key Basic Special Funds (2012CB1143001), 2015 Henan outstanding talent project (154200510006), and the Basic and Advanced Research Project of Chongqing (cstc2014jcyjA80032). The funding bodies had no role in the design of the study and collection, analysis and interpretation of data and in writing the manuscript.

\section{Availability of data and materials}

All data generated or analyzed during this study are included within this published article and its supplementary information files.

\section{Authors' contributions}

LX participated in design of the study, identification and classification of NBS-encoding genes, and drafted the manuscript. JL participated in design of the study, data preparation and drafted the manuscript. CW participated in protein structure and similarity analysis of NBS-encoding genes. YD participated in programming with Perl. CC participated in chromosome location and gene cluster identification. XZ participated in the sequence alignment and phylogenetic analysis. YC conceived of the study, and participated in its coordination and helped to draft the manuscript. All authors read and approved the final manuscript.

\section{Competing interests}

The authors declare that they have no competing interests.

\section{Consent for publication}

Not applicable.

\section{Ethics approval and consent to participate}

Not applicable.

\section{Publisher's Note}

Springer Nature remains neutral with regard to jurisdictional claims in published maps and institutional affiliations.

\section{Author details}

${ }^{1}$ State Key Laboratory of Cotton Biology, College of Life Science, Henan Key Laboratory of Plant Stress Biology, Henan University, Kaifeng, Henan 475004, China. ${ }^{2}$ College of Bioinformation, Chongqing University of Posts and Telecommunications, Chongqing 400065, China. ${ }^{3}$ United States Department of Agriculture, Southern Plains Agricultural Research Center, Agricultural Research Service, 2765 F \& B Rd, College Station, TX 77845, USA.

Received: 24 November 2016 Accepted: 4 April 2017

Published online: 12 April 2017

\section{References}

1. Wendel J, Brubaker C, Alvarez I, Cronn R. In: Paterson AH, editor. Genetics and genomics of cotton, vol. 3. New York: Springer; 2009. p. 3-22.

2. Li F, Fan G, Wang K, Sun F, Yuan Y, Song G, et al. Genome sequence of the cultivated cotton Gossypium arboreum. Nat Genet. 2014;46(6):567-72.

3. Pegg G, Brady B. Verticillium wilts 552. New York: CABl; 2002.

4. Khadi BM, Santhy V. In: Zehr UB, editor. Cotton: biotechnological advances. New York: Springer; 2010. p. 15-44.

5. Du W, Du X, Ma S. Progress of inheritance and molecular biology of Verticillium wilt resistance in cotton. Cotton Sci (China). 2002;14:311-7.

6. Cai Y, He X, Mo J, Su Q, Yang J, Liu J. Molecular research and genetic engineering of resistance to Verticillium wilt in cotton: a review. Afr J Biotechnol. 2009:8:7363-72.

7. Zhang J, Sanogo S, Flynn R, Baral J, Bajaj S, Hughs SE, et al. Germplasm evaluation and transfer of Verticillium wilt resistance from Pima (Gossypium barbadense) to Upland cotton (G hirsutum). Euphytica. 2011;187:147-60

8. Wilhelm S, Sagen JE, Tietz H. Resistance to Verticillium wilt in cotton: sources, techniques of identification, inheritance trends, and the resistance potential of multiple cultivars. Phytopathology. 1974;64:924-31.

9. Peng J, Qu Y, Wang L, Wang C, Hao W. A study on properties of resistance to Fusarium wilt in leaves of Sea Island cotton (Gossypium barbadense) and Upland cotton (Gossypium hirsutum L.) under different temperature conditions. Xinjiang Agric Sci (China). 2013;50(1):89-93.

10. Gu B, Ma C. Cotton breeding of resistant varieties in China. Nanjing: Jiangsu Science and Technology Press (China); 1996. p. 1-50.

11. Jones J, Dang J. The plant immune system. Nature. 2006;444:323-9.

12. Zipfel C. Pattern-recognition receptors in plant innate immunity. Curr Opin Immunol. 2008:20:10-6.

13. Sanseverino W, Hermoso A, D'Alessandro R, Vlasova A, Andolfo G, Frusciante L. PRGdb 2.0: towards a community-based database model for the analysis of R-genes in plants. Nucleic Acids Res. 2012;41(D1):D1167-71.

14. Yue JX, Meyers BC, Chen JQ, Tian D, Yang S. Tracing the origin and evolutionary history of plant nucleotide-binding site-leucine-rich repeat (NBSLRR) genes. New Phytol. 2012;193:1049-63.

15. McHale L, Tan X, Koehl P, Michelmore R. Plant NBS-LRR proteins: adaptable guards. Genome Biol. 2006;7(4):212.

16. Tameling WI, Elzinga SD, Darmin PS, Vossen JH, Takken FL, Haring MA, et al. The Tomato R Gene products $1-2$ and Mi-1 are functional ATP binding proteins with ATPase activity. Plant Cell. 2002;14:2929-39.

17. Meyers BC, Kozik A, Griego A, Kuang H, Michelmore RW. Genome-wide analysis of NBS-LRR-encoding genes in Arabidopsis. Plant Cell. 2003;15:809-34.

18. Chisholm ST, Coaker G, Day B, Staskawicz BJ. Host-microbe interactions: shaping the evolution of the plant immune response. Cell. 2006;124:803-14. 
19. Mun JH, Yu HJ, Park S, Park BS. Genome-wide identification of NBS encoding resistance genes in Brassica rapa. Mol Genet Genomics. 2009;282:617-31.

20. Wang K, Wang Z, Li F, Ye W, Wang J, Song G, et al. The draft genome of a diploid cotton Gossypium raimondii. Nat Genet. 2012;44(10):1098-103.

21. Li F, Fan G, Lu C, Xiao G, Zou C, Kohel RJ, et al. Genome sequence of cultivated upland cotton (Gossypium hirsutum TM-1) provides insights into genome evolution. Nat Biotechnol. 2015;33(5):524-30.

22. Yuan D, Tang Z, Wang M, Gao W, Tu L, Jin X, et al. The genome sequence of Sea-Island cotton (Gossypium barbadense) provides insights into the allopolyploidization and development of superior spinnable fibres. Sci Rep. 2015;5:17662

23. Yang S, Zhang X, Yue JX, Tian D, Chen JQ. Recent duplications dominate NBS-encoding gene expansion in two woody species. Mol Genet Genomics. 2008;280(3):187-98.

24. Jia $Y$, Yuan $Y$, Zhang $Y$, Yang S, Zhang X. Extreme expansion of NBS-encoding genes in Rosaceae. BMC Genet. 2015;16:48.

25. Cheng $Y$, Li X, Jiang H, Ma W, Miao W, Yamada T, et al. Systematic analysis and comparison of nucleotide-binding site disease resistance genes in maize. FEBS J. 2012;279:2431-43.

26. Cheng $X$, Jiang H, Zhao $Y$, Qian $Y$, Zhu S, Cheng B, et al. A genomic analysis of disease-resistance genes encoding nucleotide binding sites in Sorghum bicolor. Genet Mol Biol. 2010;33:292-7.

27. Meyers BC, Kozik A, Griego A, Kuang H, Michelmore RW. Genomewide analysis of NBS-LRR-encoding genes in Arabidopsis. Plant Cell. 2003;5:809-34

28. Leister D. Tandem and segmental gene duplication and recombination in the evolution of plant disease resistance gene. Trends Genet. 2004;20:116-22.

29. Yang S, Feng Z, Zhang X, Jiang K, Jin X, Hang Y, et al. Genome-wide investigation on the genetic variations of rice disease resistance genes. Plant Mol Biol. 2006;62:181-93.

30. Li J, Ding J, Zhang W, Zhang Y, Tang P, Chen JQ, et al. Unique evolutionary pattern of numbers of gramineous NBS-LRR genes. Mol Genet Genomics. 2010;283:427-38.

31. Friedman A, Baker B. The evolution of resistance genes in multi-protein plant resistance systems. Curr Opin Genet Dev. 2007;17:493-9.

32. Zhou T, Wang Y, Chen JQ, Araki H, Jing Z, Jiang K, et al. Genome-wide identification of NBS genes in japonica rice reveals significant expansion of divergent non-TIR NBS-LRR genes. Mol Genet Genomics. 2004;271:402-15.

33. Holub E. The arms race is ancient history in Arabidopsis, the wildflower. Nat Rev Genet. 2001;2:516-27.

34. Guo YL, Fitz J, Schneeberger K, Ossowski S, Cao J, Weigel D. Genome-wide comparison of nucleotide-binding site-leucine-rich repeat-encoding genes in Arabidopsis. Plant Physiol. 2011;157(2):757-69.

35. Ameline-Torregrosa C, Wang B, O'Bleness M. Identification and characterization of nucleotide-binding site-leucine-rich repeat genes in the model plant Medicago truncatula. Plant Physiol. 2008;146:5-21.

36. Wei H, Li W, Sun X, Zhu S, Zhu J. Systematic analysis and comparison of nucleotide-binding site disease resistance genes in a diploid cotton Gossypium raimondii. PLoS One. 2013:8(8):e68435.

37. Khan AM, Khan AA, Azhar MT, Amrao L, Cheema HM. Comparative analysis of resistance gene analogues encoding NBS-LRR domains in cotton. J Sci Food Agric. 2016;96(2):530-8.

38. He L, Du C, Covaleda L, Xu Z, Robinson AF, Yu JZ, et al. Cloning, characterization, and evolution of the NBS-LRR-encoding resistance gene analogue family in polyploid cotton (Gossypium hirsutum L.). Mol Plant Microbe Interact. 2004;17(11):1234-41.

39. Zhang T, Hu Y, Jiang W, Fang L, Guan X, Chen J, et al. Sequencing of allotetraploid cotton (Gossypium hirsutum L. acc. TM-1) provides a resource for fiber improvement. Nat Biotechnol. 2015;33(5):531-7.

40. Liu X, Zhao B, Zheng HJ, Hu Y, Lu G, Yang CQ, et al. Gossypium barbadense genome sequence provides insight into the evolution of extra-long staple fiber andspecialized metabolites. Sci Rep. 2015:5:14139.

41. Finn RD, Clements J, Arndt W, Miller BL, Wheeler TJ, Schreiber F, et al. HMMER web server: 2015 update. Nucleic Acids Res. 2015;43(W1):W30-8.

42. Thompson J, Higgins D, Gibson T. CLUSTAL W: improving the sensitivity of progressive multiple sequence alignment through sequence weighting, position-specific gap penalties and weight matrix choice. Nucleic Acids Res. 1994;22:4673-80.
43. Wang Y, Tang H, Debarry JD, Tan X, Li J, Wang X, et al. MCScanX: a toolkit for detection and evolutionary analysis of gene synteny and collinearity. Nucleic Acids Res. 2012;40(7):e49.

44. Tamura K, Peterson D, Peterson N, Stecher G, Nei M, Kumar S. MEGA5: molecular evolutionary genetics analysis using maximum likelihood, evolutionary distance, and maximum parsimony methods. Mol Biol Evol. 2011;28:2731-9.

\section{Submit your next manuscript to BioMed Central and we will help you at every step:}

- We accept pre-submission inquiries

- Our selector tool helps you to find the most relevant journal

- We provide round the clock customer support

- Convenient online submission

- Thorough peer review

- Inclusion in PubMed and all major indexing services

- Maximum visibility for your research

Submit your manuscript at www.biomedcentral.com/submit
C) Biomed Central 